INPLASY

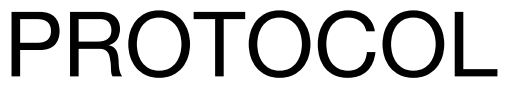

To cite: Luo et al. Speech and language features applied to the identification of patients with cognitive impairment: A systematic review and metaanalysis. Inplasy protocol

202230022. doi:

10.37766/inplasy2022.3.0022

Received: 05 March 2022

Published: 05 March 2022

Corresponding author:

Xiaqin Luo

779338013@qq.com

Author Affiliation:

Jiangsu Normal University

Support: Provincial Incubation Program.

Review Stage at time of this submission: Data extraction.

\section{Speech and language features applied to the identification of patients with cognitive impairment: A systematic review and meta-analysis}

Luo, X1; Chen, L².

Review question / Objective: Language screening is very promising as an easily accessible, low-cost and highefficiency tool. In this paper, we explore the identification role played by speech and language features in the diagnosis of $\mathrm{AD}$ and $\mathrm{MCl}$ through systematic analysis.

Information sources: We will primarily search PubMed, Web of Science, Springer, studies regardless of publication date. for the missing information we will contact the author. Additionally, the bibliographies of relevant articles were scanned for further suitable literature.

INPLASY registration number: This protocol was registered with the International Platform of Registered Systematic Review and Meta-Analysis Protocols (INPLASY) on 05 March 2022 and was last updated on 05 March 2022 (registration number INPLASY202230022).

Conflicts of interest:

None declared.

\section{INTRODUCTION}

Review question / Objective: Language screening is very promising as an easily accessible, low-cost and high-efficiency tool. In this paper, we explore the identification role played by speech and language features in the diagnosis of $A D$ and $\mathrm{MCl}$ through systematic analysis.

Condition being studied: As the global population ages, cognitive impairment is a common symptom in the elderly population, with severe cases referred to as dementia. Studies have shown that the prevalence of dementia is closely related to age, with Alzheimer's disease in particular having the highest prevalence and mild cognitive impairment being an intermediate state between aging and dementia. Early detection, early diagnosis and early treatment have become a global consensus for dementia prevention and treatment. Language impairment is a sensitive 
manifestation of impairment in higher brain functions, and by translating these significant speech changes that can be observed in patients with cognitive impairment into simple, specific language features, we can help to detect this cognitive decline earlier. As the global population ages, cognitive impairment is a common symptom in the elderly population, with severe cases referred to as dementia. Studies have shown that the prevalence of dementia is closely related to age, with Alzheimer's disease in particular having the highest prevalence and mild cognitive impairment being an intermediate state between aging and dementia. Language impairment is a sensitive manifestation of impairment in higher brain functions, and by translating these significant speech changes that can be observed in patients with cognitive impairment into simple, specific language features, we can help to detect this cognitive decline earlier.

\section{METHODS}

Participant or population: Inclusion criteria: 1. Diagnosis of Alzheimer's disease or mild cognitive impairment; 2 . The study is an observational study; 3 . The study should use speech and language features to screen $A D / M C l$ patients and healthy controls; 4. The study should provide detailed demographic information or inform whether this information matches. Reviews, editorials and case reports are not included.

Intervention: Not applicable.

Comparator: Not applicable.

Study designs to be included: Observational study.

Eligibility criteria: Inclusion criteria: 1. Diagnosis of Alzheimer's disease or mild cognitive impairment; 2 . The study is an observational study; 3 . The study should use speech and language features to screen $A D / M C l$ patients and healthy controls; 4. The study should provide detailed demographic information or inform whether this information matches.

Information sources: We will primarily search PubMed, Web of Science, Springer, studies regardless of publication date. for the missing information we will contact the author. Additionally, the bibliographies of relevant articles were scanned for further suitable literature.

Main outcome(s): Sensitivity, Specificity or Precision.

Quality assessment / Risk of bias analysis: Two authors will assess the risk of bias with the Cochrane Collaboration's tool for risk of bias assessment for all included studies.

Strategy of data synthesis: Heterogeneity between studies was detected by Cochran's $Q$-test with $P<0.05$ as a significance level, and quantitatively measured through $I^{2}$ statistic. A fixed effect model (FEM) was applied to perform the meta-analysis if the P-value of Cochran Qtests was more than 0.05 , otherwise, random effects model was utilized.

Subgroup analysis: Subgroup analysis based on study designs, otherwise Subgroup analyses will be performed (if data can be pooled) to assess the effect of age and education on cognitive impairment in screening for language characteristics.

Sensitivity analysis: After deleting any one of them, the combined results of the remaining papers were not significantly different from those without deletion, which means that the sensitivity analysis was passed.

Country(ies) involved: China.

Keywords: Speech; Language; Feature; Alzheimer's disease; Mild cognitive impairment.

Contributions of each author:

Author 1 - Xiaqin Luo.

Author 2 - Luyao Chen. 